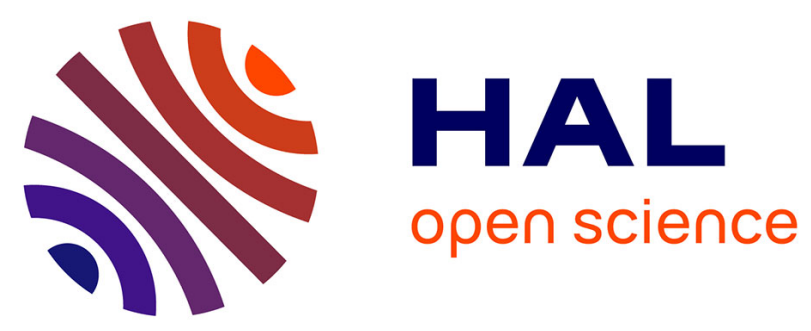

\title{
A flexible distance for the spatial placement in a multimedia document
}

Azze-Eddine Maredj, Tonkin Nourreddine, Madjid Sadallah, Zaia Alimazighi

\section{To cite this version:}

Azze-Eddine Maredj, Tonkin Nourreddine, Madjid Sadallah, Zaia Alimazighi. A flexible distance for the spatial placement in a multimedia document. Communication Technologies: from Theory to Applications (ICTTA), Apr 2008, Damascus, Syria. 10.1109/ICTTA.2008.4530102 . hal-02912372

\section{HAL Id: hal-02912372 https://hal.science/hal-02912372}

Submitted on 5 Aug 2020

HAL is a multi-disciplinary open access archive for the deposit and dissemination of scientific research documents, whether they are published or not. The documents may come from teaching and research institutions in France or abroad, or from public or private research centers.
L'archive ouverte pluridisciplinaire HAL, est destinée au dépôt et à la diffusion de documents scientifiques de niveau recherche, publiés ou non, émanant des établissements d'enseignement et de recherche français ou étrangers, des laboratoires publics ou privés. 


\section{A flexible distance for the spatial placement in a multimedia document}

\author{
Azze-Eddine Maredj, Tonkin Nourreddine, Madjid \\ Sadallah \\ Théorie et ingénierie des systèmes informatiques \\ Research Center on Scientific and Technical Information, \\ Alger, Algérie \\ amaredj@cerist.dz,ntonkin@cerist.dz,msadallah@cerist.dz
}

\author{
Zaia Alimazighi \\ Laboratoire des systèmes informatiques \\ University of science and technology Houari Boumèdiene, \\ Alger, Algérie \\ Alimazighi@wissal.dz
}

\begin{abstract}
In the multimedia documents authoring systems the management of spatial and temporal inter-objects relations is the most delicate task. Spatial relations management refers to the appropriate means to express relations between the document objects and guarantee their consistency. Usually it is represented by spatial model which performances depend on its expressivity degree, on its positioning precision and on the ability to express a desired overlap. One of the most important factor that affects performnaces is the distance associated to the relations. To enhance the expressivity and precision degrees and to allow the specification of desired overlap, we introduce, in this paper, the flexible distance concept
\end{abstract}

Keywords- Multimedia documents authoring, spatial relation, spatial model, flexible distance

\section{INTRODUCTION}

According to the author's requirements, the spatial presentation process aims to place the document objects displayed at the same time on a finite space (e.g. the screen) while checking and maintaining the objects spatial relations consistency along the multimedia document lifetime.

The spatial positioning management can be performed using a spatial model that intends two main features of multimedia design: firstly, providing the author a specification language for defining spatial relations between objects and secondly, the implementation of checking mechanisms for spatial consistency [1] [2].

In the The multimedia documents authoring systems, the spatial model performances depend on its expressivity degree: the author may build complex scenarios. On its positioning precision: the author must be able to specify the exact placement of an object. And on the ability to express a desired spatial overlap: a spatial overlap must not be automatically considered as inconsistency as it can be required by the author (images incrustation, text scroll on an image, etc.) [3].

One of the most important factor that affects the performances of a spatial model, except the relations choice, is the definition of the associated distance to the relations. Indeed, a relation with a distance is more expressive and precise than the same relation without it. Hence, and in order to increase the degree of expressivity, to enhance the precision and to allow the specification of desired overlap, we introduce, in this paper, the flexible distance concept.

The remainder of this paper is organized in three sections. In the second section, some spatial models are presented and discussed. We describe, in the third section, the concept of the flexible distance and we show its contribution in the spatial model performances. The fourth section concludes this paper.

\section{SPATIAL MODELS}

In general, two spatial positioning approaches can be distinguished: the absolute positioning and the relative positioning [4]. With the absolute positioning models, the object is placed on the presentation area at a fixed absolute position specified by a coordinate pair. A more flexible way to define the spatial positioning of objects is the use of relative positioning by specification of spatial relations between these objects [5][6][7][8]. In a two-dimension space, this approach defines several spatial presentation models: interval-based models and region-based models [9].

\section{A. Interval-Based Approach}

In this approach, an object is seen as the composition of intervals corresponding to its projections on the $\mathrm{X}$ and $\mathrm{Y}$ axis. Thus, an object is represented by the smallest rectangular box that contains it (MBR : Minimum Bounding Rectangle). The presentation composition consists on determining the absolute and/or relative objects positions on the $\mathrm{X}$ and $\mathrm{Y}$ axis. The relative positions are expressed using the Allen interval-based relations defined by the combination of two intervals on a directed straight line [10]. This set is composed of the seven basic relations (before, meet, overlap, finish, during, start and equal) and their opposites.

\section{B. Region-Based Approach}

In this approach, the objects are represented by regions such as rectangles [6] [7], circles [8] or irregular forms [5] [7]. Each region has a benchmark (e.g. its center of gravity). The 
absolute position of an object is given by its benchmark position and its relative position results from spatial relations which are classified in two types : topological relations and directional relations.

Topological relations describe concepts of neighborhood, incidence and overlap and stay invariant under transformations such as scaling and rotation. In the model proposed by M.J. Egenhofer [11], 8 topological relations between two region objects have been defined: disjoint, touch, overlap, cover, covered by, contain, inside and equal.

Directional relations describe the order of objects in a definite space (e.g. north, south). The Papadias and al. [7] model in the field of geographic information systems comprises a set of 9 relations considered sufficient to express any directional relation between two regions: north, south, east, west, northeast, northwest, southeast, southwest and sameposition.

Both the two approaches have an acceptable expressivity degree and offer means to express desired overlap. However, the lack of a distance reduces considerably the object placement precision. The generalized relations (directional and topological) are not sufficient to represent all the different semantics of objects composition, since they do not convey the necessary information. For instance we want to present two objects $A$ and $B$ where $B$ is on the right side of A with a gap of 8 units; the topological relation "disjoint" does not capture the relative position (right) and the directional relation "east" does not indicate the distance between the two objects.

Moreover, considering an object as a point (e.g. its gravity center), with the region-base approaches, may induce a positioning other than expected by the relation semantic. For example, the relation $B$ east $\mathrm{A}$ which means that the object $\mathrm{B}$ must be placed on the right of the object $A$, can lead to a non desired overlap of the object A by the object B (figure 1.a) and even to a placement other than the one given by the relation semantic, as shown in figure 1.b where the object $A$ is contained by the object $\mathrm{B}$, although the benchmark of $\mathrm{B}$ is on the right of the benchmark of A.

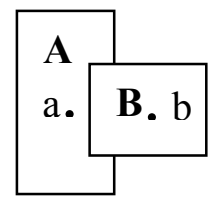

(a)

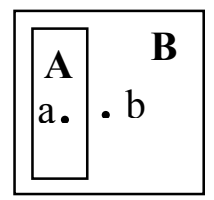

(b)
Figure 1. Generation of non- specified placements

One solution to this problem would be to consider and calculate the distance between the benchmarks. This may be very complex particularly with irregular regions as illustrated in the figure 2 .

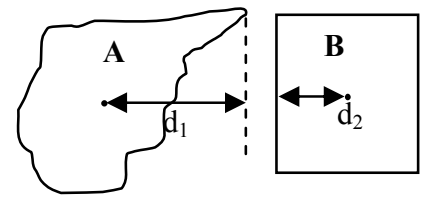

Figure 2. Distance calculation complexity

\section{FLEXIBLE DISTANCE}

Before defining the flexible distance concept, we start by presenting the retained spatial representation model of an object and its neighborhood definition.

\section{A. The Object Spatial Representation Modeling}

In the multimedia documents authoring systems, the handled objects, except the audio, have a rectangular form. Hence, each object can be represented by a box which corresponds to its minimal container rectangle [12]. In order to simplify the relations specification and the spatial representation of an object, we take into account only its higher left and lower right angles coordinates (figure3). Indeed, to specify an object $\mathrm{B}$ is at the right side of an object $\mathrm{A}$, we have just to require the right edge of $B$ to be at the right side of the left edge of A.

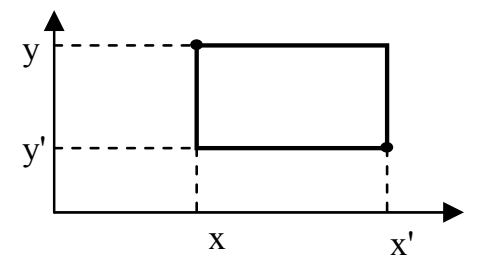

Figure 3. Object spatial representation

\section{B. The Object Neighborhood Modeling}

The neighborhood definition is an important factor for the placement precision and expressivity degrees. The aims are to allow object placement in the entire related object neighborhood witch must be partitioned and oriented. However, we choose the A.U. Frank's model [13] that is considered enough complete for rectangular object neighborhood representation. An object neighborhood is divided into eight cells (north, south, east, west, north-east, north-west, south-east and south-west), as shown in figure 4.

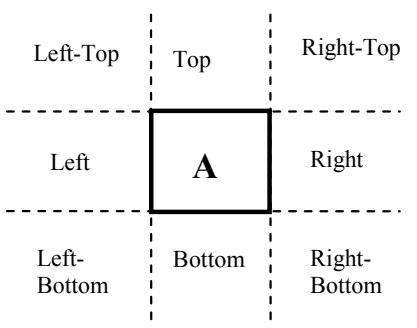

Figure 4. Object's neighbourhood.

\section{Flexible Distance}

A distance is the gap between two objects related by a spatial relation. when it is defined in a spatial model, the distance improves greatly the relations expressivity and allow a better placement precision. With the existing models, when it is defined, the distance is generally given as a fixed value. 
To enhance the expressivity and the placement precision degrees, we propose the flexible distance concept $d_{f}$. It is defined as the gap between the coordinates of the higher left and the lower right angles of two related object. Its value is taken in an interval: $d_{f} \in\left[\delta^{l}, \delta^{2}\right], \delta^{l}<\delta^{2}$. The interval edges, when specified, are positive, negative or null, otherwise, not specified, they are represented by the '-' character.

Taking into account the spatial representation, we define, at most, two flexible distances for a relation (figure 5): $d_{f x} \in\left[\delta_{l}{ }^{l}\right.$, $\left.\delta_{1}^{2}\right]$ and $d_{f y} \in\left[\delta_{2}{ }^{l}, \delta_{2}^{2}\right]$, respectively the flexible distance on $\mathrm{X}$ and $\mathrm{Y}$ axis.

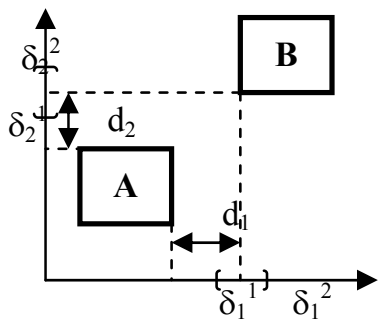

Figure 5. Flexible distance.

\section{Expressivity Model Enhancement}

With the flexible distance concept, the author can specify relations using the at nearest and at farthest expressions, that are not defined in any existing model. The TABLE I summarizes the whole expressions given by the values of the interval edges.

TABLE I. INTERPRETATION OF VALUES INTERVAL EDGES

\begin{tabular}{|l|l|}
\hline \multicolumn{1}{|c|}{ Interval } & \multicolumn{1}{c|}{ Interpretation } \\
\hline$[\delta 1, \delta 2]$ & At nearest $\delta^{1}$ units and at farthest $\delta^{2}$ units; $\delta^{1}<\delta^{2}$. \\
\hline$[-, \delta]$ & At farthest $\delta$ units. \\
\hline$[\delta,-]$ & At nearest $\delta$ units. \\
\hline$[\delta, \delta]$ & Exactly $\delta$ units. \\
\hline$[-,-]$ & Non specified distance. \\
\hline
\end{tabular}

To illustrate the expressivity enhancement, let's compare the specification number of the relation $B$ left $A$ in the fixed distance and flexible distance cases.

With a fixed distance, we have three possible specifications:

- B left (d) A: B is exactly $d$ units to the left of A.

- $\quad$ B left (0) A: The right edge of B is aligned with the left edge of $A$.

- $\quad$ B left (-) A : B is at the left of A.

With the flexible distance, six specifications are possible:

- B left $\left(\left[\delta^{1}, \delta^{2}\right]\right)$ A: B is at nearest $\delta^{1}$ units and at farthest $\delta^{2}$ from the left of $A$.

- $\quad$ B left $([\delta, \delta])$ A: B is exactly $\delta$ units at the left of A.
- $\quad \mathrm{B}$ left $([\delta,-]) \mathrm{A}: \mathrm{B}$ is at nearest $\delta$ units from the left of A.

- $\quad$ B left $([-, \delta]) \mathrm{A}: \mathrm{B}$ is at farther $\delta$ units from the left of A.

- $\quad$ B left $([0,0]) \mathrm{A}$ : The right edge of $\mathrm{B}$ is aligned to the left edge of $A$.

- $\quad$ B left $([-,-]) \mathrm{A}: \mathrm{B}$ is at the left of A.

\section{E. Precision Of The Placements}

With the flexible distance concept, the author has the possibilitie to specifie any desired palcement. Quantitatively, the author may either specify the distances between the related objects, restrict them in an interval or not specify any distance when there's no placement preference. Qualitatively, with the neighborhood partition and the negative distance definition, the space of placement wraps all the object neighborhood, even the placement on two cells is possible. The relation $B$ right_top $\left(d_{f}\right.$, $\left.d_{f y}\right) A$, with $d_{f y}<0$, for example, allows placing the object $\mathrm{B}$ on two cells of the A object neighborhood (figure 6).

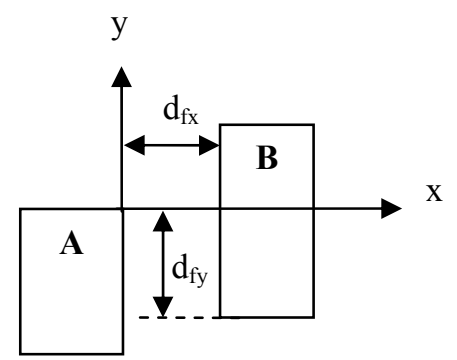

Figure 6. Object placement on two cells

\section{F. Overlap Specification}

As seen, In the multimedia documents authoring systems, the spatial overlaps should not be automatically considered as an inconsistency. The use of a negative distance allows to express any desired overlap without defining any specific relation, what optimise the relations number of the model. For example The relation $B$ right $([-10,-10]) A$, lets $B$ to cover $A$ by 10 units from the right (figure 7 ).

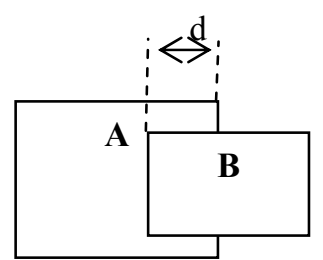

Figure 7. Desired overlap specification

However, for the relation $\mathbf{B} R \mathbf{A}$, where $\left|d_{f x}\right| \geq \mathbf{A}$.width and $\left|d_{f y}\right| \geq \mathbf{A}$.height, the placement of $\mathbf{B}$ will differ from the one expected by the author. For example, with the relation B left_bottom $\left(d_{f x}, d_{f y}\right)$ A, represented in the figure 8.a, if $\left|d_{f x}\right| \geq$ A.width and $\left|d_{f y}\right| \geq \mathbf{A}$.height, we will obtain the configuration 
ed by the figure $8 . \mathrm{b}$ that corresponds to the relation $\mathbf{B}$ $\left(d_{f x}^{\prime}, d_{f y}^{\prime}\right) \mathbf{A}$.

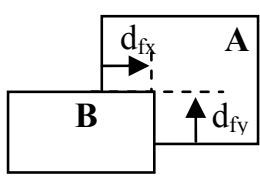

(a)

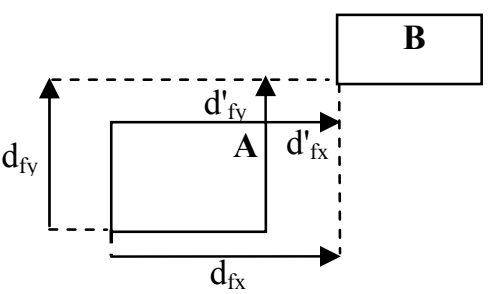

(b)
Figure 8. Placement other than specified

One solution to this problem is to pose the following constraints :

$\left|d_{f x}\right| \leq$ reference. width and $\left|d_{f y}\right| \leq$ reference. height, where reference is the object at the right of the relation.

\section{CONCLUSION}

In this paper, we propose a new distance definition to the multimedia documents authoring systems. The concept of flexible distance allows the use of at nearest and at farther expressions, that are not defined in any existing model. In addition, combined with the neighborhood definition, it gives to the relations not only a very high degree of precision but also the possibility to place an object on two different cells. Without any specific relation, a desired overlap may be expressed using a negative distance. Finally, an object is likely more to be placed when its distance is from an interval: the restriction of placement is less strong.

\section{REFERENCES}

[1] Perez-Luque M. J., Little T. D. C., ”A Temporal Reference Framework for Multimedia Synchronisation", IEEE Journal on Selected Areas in Communications, vol. 14, no. 1, pp. 36-51, 1996.

[2] Layaida N., "Madeus: un système d'édtion et de présentation de documents structurés multimédia". Thèse $\mathrm{PhD}$, Université Josèphe Fourier, Grenoble-France, Juin 1997.

[3] Maredj A., Alimazighi Z., Hammour A. "Contraintes disjonctives pour la gestion du recouvrement spatial dans les documents multimédia", Information-Interaction- intelligence, A Journal in Information Engineering Sciences, Vol. 6, no1, pp 9-29, 2006.

[4] Boll S., Klas W. and Westermann U., "A Comparison of Multimedia Document Models Concerning Advanced Requirements", Technical Report 99-01, Databases and Information Systems, Computer Science Department, Ulm, Germany, 1999.

[5] Egenhofer M.J. "Spatial SQL: a query and presentation language", IEEE Transaction on knowledge and data engineering. vol. 6, no. 1, pp. 8695, 1994.

[6] Papadias D., Theodoridis Y., Sellis T. and Egenhofer M., "Topological relations in the world of minimum bounding rectangles: A study with $\mathrm{R}$ trees". Proceedings of the 1995 ACM SIGMOD International Conference on Management of Data, Vol. 24 , no., pp. 92 - 103, 1995.

[7] Papadias D. and Theodoridis Y.. "Spatial relations, minimum bounding rectangles, and spatial data structures", International journal of geographic information science, vol. 11 no. 2, 1997.

[8] Safar M. and Shahabi C., "2D topological and direction relations in the world of minimum bounding circles", in Proceeding of IDEAS International database engineering and application symposium, Montreal, Canada, pp. 239 - 247, 1999.

[9] Zechinilli Martini J.L., Construction et manipulation de présentations spatio-temporelles multimédias à partir de serveurs d'éléments repartis : Application aux données sur le web. Thèse de doctorat. Université Joseph Fourier 2001.

[10] Allen J.F., "Maintaining knowledge about temporal intervals", Communication of the ACM, vol. 26, no 11, pp. 832 - 843, 1983.

[11] Egenhofer M.J., Franzosa R., "Point-Set Topological Spatial Relations", International Journal of Geographical Information Systems, vol. 5, no. 2, pp 161-174, March 1991.

[12] Maredj A., Hammour A., Ider D., 'Une approche basée sur le simplexe pour le formatage spatial de documents multimédia interactifs' in Conférence Internationale: Sciences Electroniques, Technologies de l'information et des Télécommunications SETIT04 Sousse, Tunisie. 2004.

[13] Frank, A. U. "Qualitative Spatial Reasoning: Cardinal Directions as an Example", International journal of geographical information science, vol. 10, no. 3, pp. 269-290, 1996. 